\title{
Coupled Networks of Parvalbumin-Immunoreactive Interneurons in the Rat Basolateral Amygdala
}

\author{
Jay F. Muller, Franco Mascagni, and Alexander J. McDonald \\ Department of Pharmacology, Physiology, and Neuroscience, University of South Carolina School of Medicine, Columbia, South Carolina 29208
}

\begin{abstract}
Recent studies indicate that the basolateral amygdala exhibits fast rhythmic oscillations during emotional arousal, but the neuronal mechanisms underlying this activity are not known. Similar oscillations in the cerebral cortex are generated by a network of parvalbumin (PV)-immunoreactive interneurons interconnected by chemical synapses and dendritic gap junctions. The present immunoelectron microscopic study revealed that the basolateral amygdalar nucleus (BLa) contains a network of parvalbumin-immunoreactive $(\mathrm{PV}+)$ interneurons interconnected by chemical synapses, dendritic gap junctions, and axonal gap junctions. Twenty percent of synapses onto $\mathrm{PV}+$ neurons were formed by $\mathrm{PV}+$ axon terminals. All of these $\mathrm{PV}+$ synapses were symmetrical. $\mathrm{PV}+$ perikarya exhibited the greatest incidence of PV + synapses (30\%), with lower percentages associated with PV + dendrites (15\%) and spines (25\%). These synapses comprised half of all symmetrical synapses formed with PV + cells. A total of 18 dendrodendritic gap junctions between PV + neurons were observed, mostly involving secondary and more distal dendrites $(0.5-1.0 \mu \mathrm{m}$ thick). Dendritic gap junctions were often in close proximity to $\mathrm{PV}+$ chemical synapses. Six gap junctions were observed between PV + axon terminals. In most cases, one or both of these terminals formed synapses with the perikarya of principal neurons. This is the first study to describe dendritic gap junctions interconnecting $\mathrm{PV}+$ interneurons in the basolateral amygdala. It also provides the first documentation of gap junctions between interneuronal axon terminals in the mammalian forebrain. These data provide the anatomical basis for a PV+ network that may play a role in the generation of rhythmic oscillations in the BLa during emotional arousal.
\end{abstract}

Key words: GABAergic interneurons; chemical synapses; dendritic gap junctions; axonal gap junctions; oscillations; electron microscopy

\section{Introduction}

During the last decade, it has become evident that the functional activity of many brain areas is dependent on fast synchronous oscillations of large populations of interconnected neurons (Singer, 1993). Several different rhythms have been observed both within and across different cortical areas, including theta $(4-10 \mathrm{~Hz})$, gamma $(30-100 \mathrm{~Hz})$, and ultrafast $(\sim 200 \mathrm{~Hz})$ oscillations. Theta oscillations, with superimposed gamma oscillations, are frequently seen in the hippocampus and entorhinal cortex during attentive arousal and exploratory behavior (Buzsaki, 2002). Synchronous gamma oscillations are also exhibited by neuronal assemblies in separate visual cortical areas processing different features of the same visual stimulus in somatosensory and motor cortices during a reaching task and in the frontal cortex in a delayed matching to sample task (Singer, 1993). Synchronous firing of interconnected neuronal assemblies permits temporal coding of information and promotes synaptic plasticity (Singer, 1993; Buzsaki, 2002). Thus, synchronous rhythmic activity appears to be critical for a wide variety of cortical functions, including higher-cognitive activities.

Received March 7, 2005; revised June 30, 2005; accepted June 30, 2005.

This work was supported by National Institutes of Health Grant NS38998. We thank Dr. Kenneth Baimbridge (University of British Columbia) for his generous gift of the antibody to parvalbumin.

Correspondence should be addressed to Alexander J. McDonald, Department of Pharmacology, Physiology, and Neuroscience, University of South Carolina School of Medicine, Columbia, SC 29208. E-mail:mcdonald@med.sc.edu. DOI:10.1523/JNEUROSCI.0899-05.2005

Copyright $\odot 2005$ Society for Neuroscience $\quad$ 0270-6474/05/257366-11\$15.00/0
Cortical interneurons, which use GABA as an inhibitory neurotransmitter, play an important role in the generation and maintenance of synchronous oscillations in the theta, gamma, and ultrafast bands (Freund and Buzsáki, 1996). Of particular interest is a subpopulation of interneurons that contain the calcium-binding protein parvalbumin (PV). Most cortical parvalbumin-immunoreactive $(\mathrm{PV}+)$ neurons are basket cells or chandelier cells that can entrain the firing of neighboring pyramidal cells via a robust, inhibitory, perisomatic innervation (Freund and Buzsáki, 1996). Generation of synchronous oscillations in networks of PV + neurons is dependent on the interconnections of these neurons by both GABAergic chemical synapses and electrical synapses (Gibson et al., 1999; Tamas et al., 2000; Galarreta and Hestrin, 2001). Electrical coupling of cortical PV+ interneurons is mediated by gap junctions formed by channels that connect the interiors of apposed dendrites and/or somata (Tamas et al., 2000).

The basolateral amygdala is a nuclear complex of the tempo$\mathrm{ral} /$ piriform lobe that plays a critical role in emotional behavior and learning, especially related to fear and anxiety (Aggleton, 1992, 2000; Shinnick-Gallagher et al., 2003). Recent studies have demonstrated that prominent theta and gamma oscillations occur in the amygdala during emotional arousal (Paré and Collins, 2000; Oya et al., 2002; Paré et al., 2002). Additional electrophysiological investigations have recorded neuronal activity in the basolateral amygdala at theta (Paré and Gaudreau, 1996), gamma (Collins et al., 2001), and ultrafast frequencies (Ponomarenko et 
al., 2003). Because the basolateral amygdala is a cortical-like structure exhibiting pyramidal-like projection neurons and GABAergic interneurons (McDonald, 1992), including PV+ basket cells and chandelier cells (Pitkänen and Amaral, 1993; McDonald and Betette, 2001), it is of interest to determine whether there is a network of PV + interneurons interconnected by chemical synapses and gap junctions that may be correlated with the oscillations in this region. The present electron microscopic investigation provides evidence for both types of connections between $\mathrm{PV}+$ interneurons in the basolateral amygdala. It also documents for the first time gap junctions between interneuronal axon terminals in the mammalian forebrain.

\section{Materials and Methods}

Tissue preparation. Immunocytochemical localization of parvalbumin, for ultrastructural analysis of contacts between parvalbuminimmunoreactive $(\mathrm{PV}+)$ neurons, was performed in adult male Sprague Dawley rats (250-350 g; Harlan, Indianapolis, IN). All experiments were performed in accordance with the National Institutes of Health Guide for the Care and Use of Laboratory Animals and were approved by the University of South Carolina Institutional Animal Care and Use Committee. Rats were anesthetized with chloral hydrate $(350 \mathrm{mg} / \mathrm{kg})$ and perfused intracardially with PBS, $\mathrm{pH} 7.4$, containing $1 \%$ sodium nitrite $(50 \mathrm{ml})$ followed by one of three fixation protocols: (1) three rats were perfused with $2.0 \%$ paraformaldehyde- $3.75 \%$ acrolein in $0.1 \mathrm{M}$ phosphate buffer $(\mathrm{PB})$ and postfixed in $2 \%$ paraformaldehyde for $3 \mathrm{~h}$ at $4^{\circ} \mathrm{C}$; (2) one rat was perfused with $2 \%$ paraformaldehyde $/ 2.5 \%$ glutaraldehyde in $0.1 \mathrm{M}$ $\mathrm{PB}$ and postfixed for $1 \mathrm{~h}$ at $4^{\circ} \mathrm{C}$; and (3) one rat was perfused with $4.0 \%$ paraformaldehyde $-0.2 \%$ glutaraldehyde in $\mathrm{PB}$ and postfixed in $4.0 \%$ paraformaldehyde for $6 \mathrm{~h}$ at $4^{\circ} \mathrm{C}$. The amygdalar region in each hemisphere was sectioned individually on a vibratome at a thickness of $75 \mu \mathrm{m}$ in the coronal plane. One amygdala fixed with $2 \%$ paraformaldehyde $/ 2.5 \%$ glutaraldehyde was sagittally sectioned at $60 \mu \mathrm{m}$. Sections collected from brains fixed with acrolein or $2.5 \%$ glutaraldehyde were rinsed in $1 \%$ sodium borohydride as described previously (McDonald and Betette, 2001).

Immunocytochemistry. Selected sections through the amygdala at bregma levels, -2.1 through -3.0 (Paxinos and Watson, 1986), were processed for immunohistochemistry in wells of tissue culture plates. Antibodies were diluted in PBS containing 1\% normal goat serum. For two of the preparations fixed with acrolein, antibodies were prepared with $0.02 \%$ Triton X-100. Sections from the other preparations were cryoprotected with $30 \%$ sucrose in $\mathrm{PB}$, followed by two or three cycles of freezethaw using liquid nitrogen. Sections were incubated in rabbit anti-PV (1: 2000 to 1:3000; donated by Dr. Kenneth Baimbridge, University of British Columbia, Vancouver, British Columbia, Canada) overnight at $4^{\circ} \mathrm{C}$. Previous adsorption studies have shown that this antiserum recognizes $\mathrm{PV}$ but not other calcium-binding proteins (Conde et al., 1994).

Sections were then processed for avidin-biotin immunoperoxidase (IP) using a rabbit Vectastain ABC kit (Elite or Standard; Vector Laboratories, Burlingame, $\mathrm{CA}$ ) with 3,3'-diaminobenzidine as the chromogen, and either pre-embedding immunogold-silver (IGS) technique as described previously for electron microscopic dual localization studies (McDonald et al., 2002), or the Vector VIP (V-VIP; Vector Laboratories) substrate kit for peroxidase with a reaction product that appears purple in the light microscope and yields a particulate label in the electron microscope (Smiley et al., 1997; Van Haeften and Wouterlood, 2000). The V-VIP reaction was done according to the instructions of the kit, but the steps in the subsequent dehydration series (see below) were shortened $30-50 \%$ to minimize extraction of the purple reaction product (Lanciego et al., 1997).

After rinsing in $0.16 \mathrm{M}$ sodium cacodylate buffer, $\mathrm{pH} 7.4$, sections were postfixed in $2 \%$ osmium tetroxide for $30-45 \mathrm{~min}$, and most samples were then stained en bloc with $1 \%$ uranyl acetate in $0.1 \mathrm{M}$ acetate buffer, $\mathrm{pH}$ 5.5. Sections were dehydrated in a graded ethanol series and acetone and flat embedded in Polybed 812 (Polysciences, Warrington, PA) at $60^{\circ} \mathrm{C}$. Selected areas of the anterior subdivision of the basolateral amygdalar nucleus (BLa) were remounted onto resin blanks and thin sectioned at $60-80 \mathrm{~nm}$. Serial sections were collected on formvar-coated slot grids, stained with uranyl acetate and Luft's lead citrate, and exam-

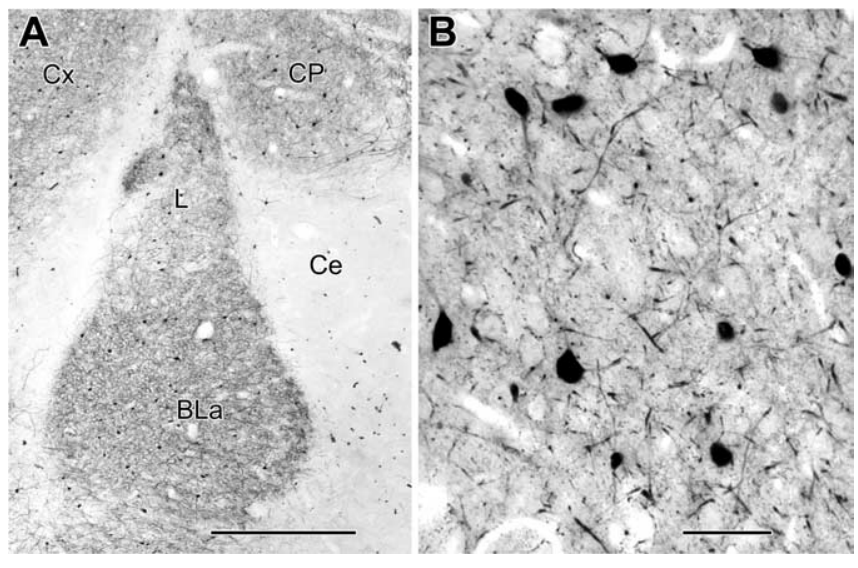

Figure 1. Light micrographs of parvalbumin immunoreactivity. A, A low-magnification micrograph of a coronal section through the rat amygdala (bregma level, -2.7 ) showing PV immunoreactivity in the BLa and adjacent regions ( $C$, central amygdalar nucleus; $L$, lateral amygdalar nucleus; $C x$, cortex; $C P$, caudatoputamen). $\boldsymbol{B}$, A higher-magnification view showing $\sim 90 \%$ of an area remounted and thin sectioned for quantitative electron microscopic analysis (see Materials and Methods). Scale bars: $\boldsymbol{A}, 500 \mu \mathrm{m} ; \boldsymbol{B}, 50 \mu \mathrm{m}$.

ined with a JEOL-200CX electron microscope (JEOL, Akishima, Japan). For quantification (see below), photographic negatives were printed, and micrographs were assembled into composites. For publication, negatives were scanned and digitized with a UMAX Power Look III flatbed scanner. Figures were then assembled and labeled, and the tonal ranges of their components were adjusted and matched using Photoshop 6.0 (Adobe Systems, San Jose, CA).

Analysis. Initial surveys were done on preparations labeled with IP and V-VIP techniques to search for possible gap junctions between PV+ profiles. Dendritic arborizations of PV + interneurons in the BLa are not stratified (see Fig. 1), as they are in cortical structures such as the hippocampus (Fukuda and Kosaka, 2000), and fixation procedures that allow good ultrastructural preservation tend to limit antibody penetration. Therefore, clear examples of adjacent immunoreactive dendrites were relatively rare (see Fig. $1 B$ ). Thus, for ultrastructural analysis, areas of the BLa were selected from flat-embedded sections in which there appeared to be adjacent immunoreactive structures. To test the possibility that the orientation or plane of sectioning might affect the coherence or lengths of labeled dendrites, sagittal as well as coronal sections containing BLa were examined.

Two samples from preparations labeled for PV using the IP procedure were chosen for quantification of synaptic and gap junctional contacts among PV+ interneurons in the BLa. One area came from a coronal section fixed with acrolein, and the other was from a sagittal section fixed with $2 \%$ paraformaldehyde $/ 2.5 \%$ glutaraldehyde. To help guarantee a good sample of all synaptic compartments, areas were chosen that had a relatively high number of strongly $\mathrm{PV}+$ perikarya. The areas were mapped using digital light micrographs from a CCD camera (QImaging MicroPublisher, Burnaby, British Columbia, Canada) so that all of the $\mathrm{PV}+$ perikarya could be identified in the electron microscope and the areas surveyed clearly delineated. For each section sampled, photographic composites of various sizes, followed for three to four serial thin sections, recorded all $\mathrm{PV}+$ structures in these areas receiving synaptic input. PV + postsynaptic structures were identified as perikarya, axon initial segments, large-caliber dendrites ( $>1 \mu \mathrm{m}$ thick), small-caliber dendrites ( $<1 \mu \mathrm{m}$ thick), and spines using standard criteria (Peters et al., 1991). All PV-immunoreactive and unlabeled axon terminals forming synapses with these PV+ structures were counted and the type of synapse (symmetrical or asymmetrical, based on the width of the postsynaptic density) was recorded. In cases in which the synapse could not be positively identified as symmetrical or asymmetrical, it was designated as "unclassified." The latter designation was used for synapses that had postsynaptic densities of intermediate thickness, were oriented obliquely to the plane of sectioning, or had postsynaptic densities that were shrouded by peroxidase reaction product. In some instances, $\mathrm{PV}+$ axons 
were seen to synapse with the perikarya or proximal dendrites of nonimmunostained pyramidal neurons, in addition to $\mathrm{PV}+$ interneurons. These pyramidal neurons were identified on the basis of several characteristic features; they exhibited large perikarya with abundant cytoplasm and only received symmetrical axosomatic synapses (Carlsen and Heimer, 1986; Carlsen, 1988; McDonald et al., 2002).

Gap junctions were identified using standard criteria (Peters et al., 1991). These junctions are heptalaminar structures in which the plasma membranes of apposed structures are separated by a uniform, narrow gap that is significantly narrower than those exhibited by puncta adherentia and chemical synapses (Sloper and Powell, 1978; Fukuda and Kosaka, 2003). Diameters of the PV + dendrites connected by gap junctions, and the lengths of those gap junctions, were measured directly from electron micrographs. Gap junctions were measured along the gap [or central line in peroxidase preparations (Fukuda and Kosaka, 2000, 2003)] (see Results).

Although most GABAergic axons in the BLa are presumed to originate from local interneurons, there is electrophysiological evidence (Mello et al., 1992) that some may arise from GABAergic neurons in the basal forebrain that are intercalated among the cholinergic neurons. Anatomical studies in the cortex have demonstrated an analogous projection and have shown that these basal forebrain GABAergic neurons express PV and exhibit very large axonal terminals that selectively target the perikarya of $\mathrm{PV}+$ interneurons and other interneuronal subpopulations (Freund, 1989). We demonstrated recently that a similar basal forebrain $\mathrm{GABA} / \mathrm{PV}+$ projection, characterized by large $\mathrm{PV}+$ axonal varicosities (1.25-2.5 $\mu \mathrm{m}$ in diameter), is associated with the BLa (our unpublished observations). To estimate the extent to which the PV+ axon terminals synapsing with $\mathrm{PV}+$ perikarya in the present study might represent this large-terminal, basal forebrain $\mathrm{GABA} / \mathrm{PV}+$ projection instead of the smaller size $(<1.0 \mu \mathrm{m}$ in diameter) (McDonald and Betette, 2001) of most PV + terminals, the cross-sectional areas of axon terminals from the following samples were compared: (1) $\mathrm{PV}+$ terminals synapsing with $\mathrm{PV}+$ perikarya in electron micrographs; (2) PV + axonal varicosities contacting large PV-negative perikarya (presumably belonging to pyramidal cells) in photomicrographs; and (3) anterogradelabeled axonal varicosities [labeled by Phaseolus vulgaris leucoagglutinin (PHA-L) injections into the basal forebrain] (for details of the PHA-L technique, see McDonald and Mascagni, 1997) contacting PV+ perikarya in photomicrographs. Samples of PV+ axon terminals/varicosities from each of these preparations were traced from micrographs and scanned into Photoshop 6.0, and their areas were calculated using Adobe ImageJ software (Adobe Systems). Comparison of brain cross-sectional areas revealed no significant differential shrinkage among these preparations.

\section{Results}

\section{Light microscopy}

At the light microscopic level, the distribution and morphology of PV + neurons in the BLa (Fig. 1) was virtually identical to that
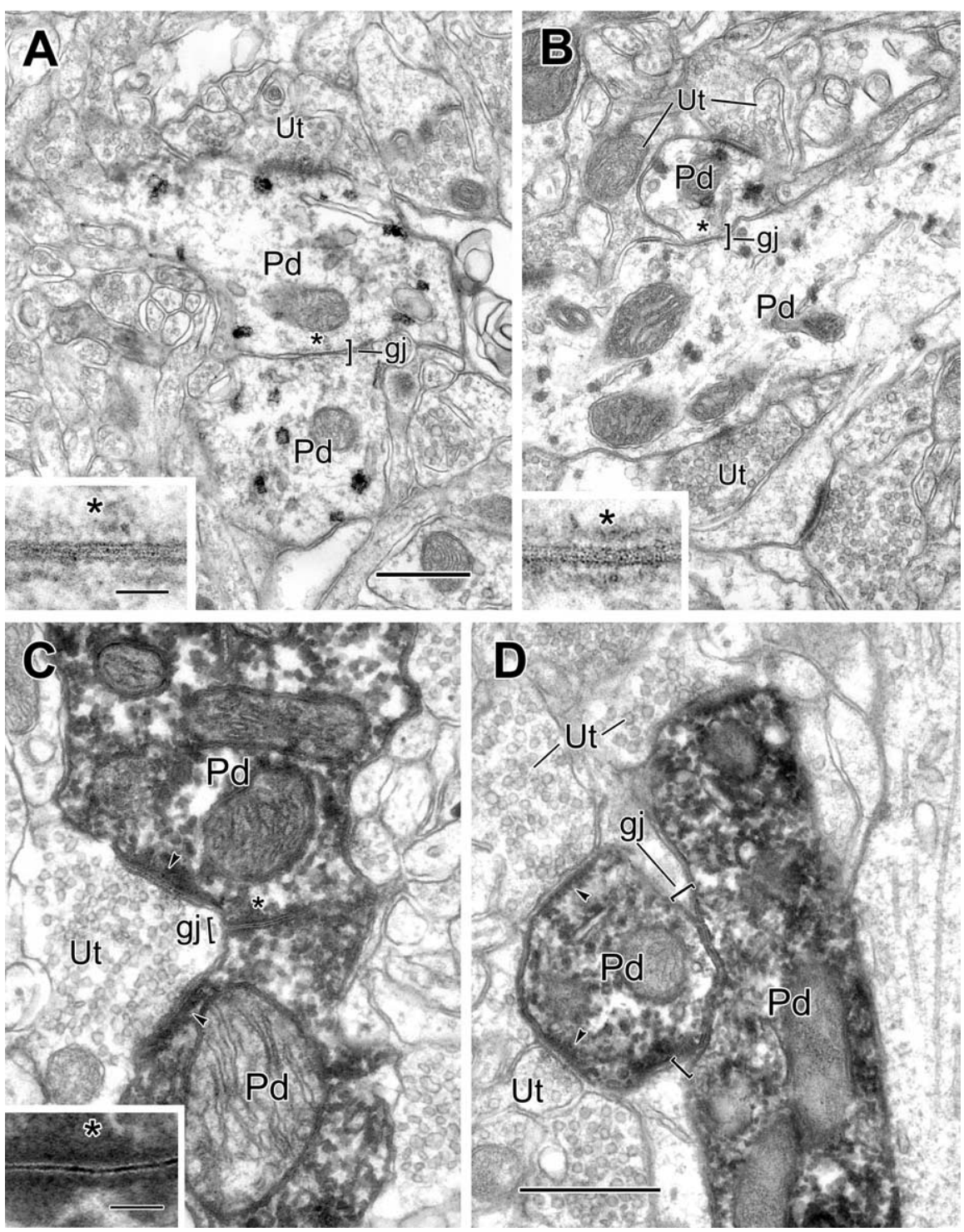

Figure 2. Electron micrographs of gap junctions between PV + dendrites in the BLa. A, A gap junction (gj; bracket), $0.22 \mu \mathrm{m}$ in length, between two PV + dendrites (Pd), labeled using the pre-embedding IGS technique. One labeled dendrite receives synaptic input from an unlabeled terminal (Ut). The asterisk indicates the site of gap junctional contact, which is enlarged in the inset. $B$, A gap junction (gj; bracket), $0.2 \mu \mathrm{m}$ in length, between two PV + dendrites (Pd), is labeled using the V-VIP substrate technique for peroxidase. The asterisk indicates the area of contact, which is enlarged in the inset. Both labeled dendrites received synaptic input from unlabeled terminals (Ut). In serial sections, one terminal (top, left) was seen to form symmetrical contacts with both dendrites, whereas the other terminals formed asymmetrical synapses. C, A gap junction (gj; bracket), $0.33 \mu \mathrm{m}$ in length, between two PV + dendrites (Pd), is labeled using the immunoperoxidase technique. The asterisk indicates the area of contact, which is enlarged in the inset. An unlabeled terminal (Ut) makes asymmetrical synaptic contact onto both labeled dendrites (arrowheads). D, A gap junction (gj; brackets), $0.5 \mu \mathrm{m}$ in length, between two PV + dendrites (Pd), is labeled using the IP technique. Both labeled dendrites receive synaptic input from unlabeled terminals (Ut; arrowheads). Scale bars: $0.5 \mu \mathrm{m}$; insets, 50 $\mathrm{nm} . \boldsymbol{B}$ is the same magnification as $\boldsymbol{A}$. $\boldsymbol{C}$ is the same magnification as $\boldsymbol{D}$.

described in previous studies (Kemppainen and Pitkänen, 2000; McDonald and Betette, 2001). PV+ somata were ovoid or multipolar and varied from $10-20 \mu \mathrm{m}$ in diameter. Most PV + neurons exhibited four to five spine-sparse dendrites that branched sparingly. Primary dendrites were usually $2-3 \mu \mathrm{m}$ thick, most secondary dendrites were $1 \mu \mathrm{m}$ thick, and distal dendrites were often $<0.5 \mu \mathrm{m}$ thick. PV + dendritic branches were randomly oriented (Fig. $1 B$ ) and occasionally appeared to contact each other at the light microscopic level. Although previous light microscopic studies reported that PV+ interneurons in the rat basolateral amygdala were "aspiny" (Kemppainen and Pitkänen, 


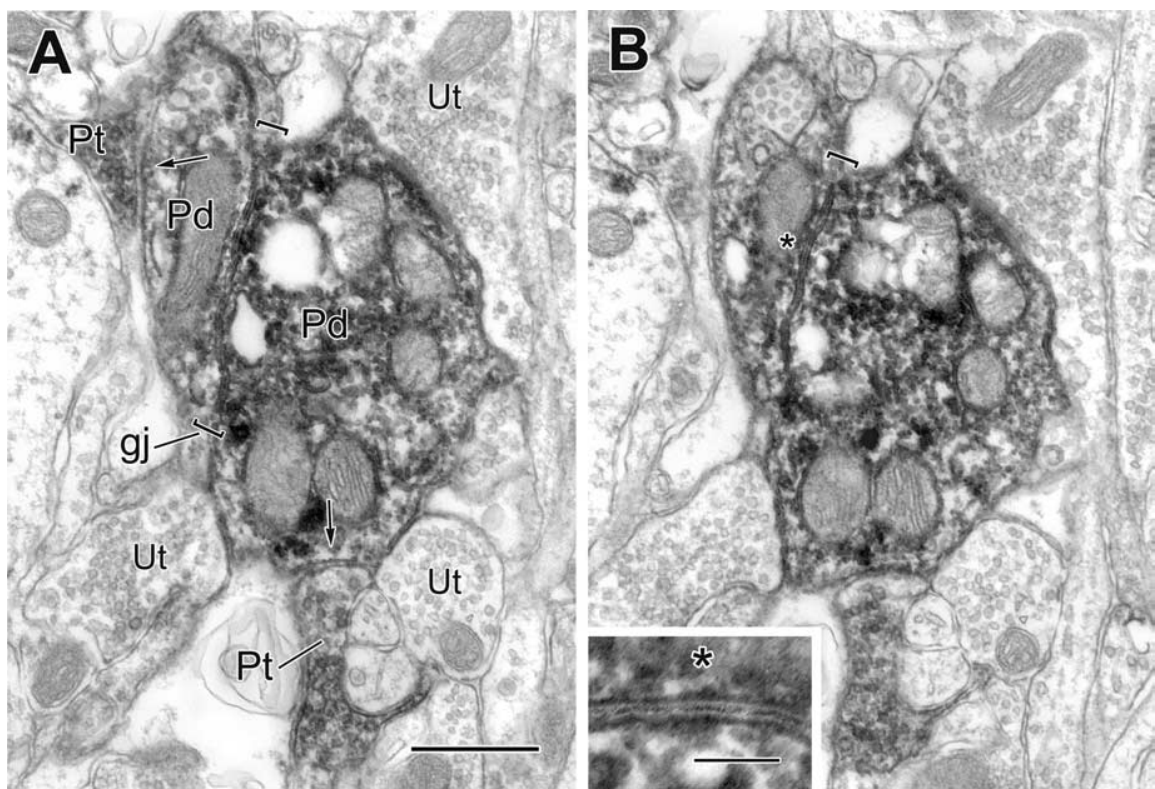

Figure 3. Two serial electron micrographs of a gap junction (gj; brackets) between two PV + dendrites (Pd), both receiving synaptic input from PV + terminals (Pt; arrows). The gap junction, followed for five serial sections, measured $0.9 \mu \mathrm{m}$ in length. The asterisk in $\boldsymbol{B}$ marks the gap junction region, which is enlarged in the inset. Unlabeled terminals (Ut) are also in contact with the larger labeled dendrite. Scale bars: $\boldsymbol{A}, \boldsymbol{B}, 0.5 \mu \mathrm{m}$; inset, $100 \mathrm{~nm}$.
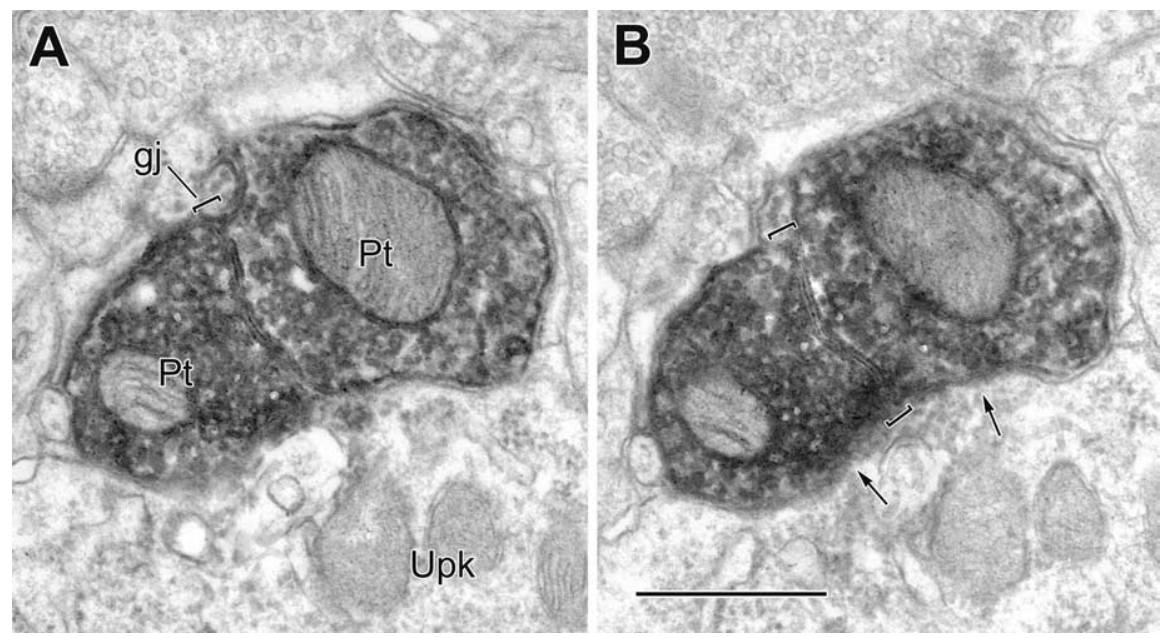

Figure 4. $\quad \boldsymbol{A}, \boldsymbol{B}$, Two serial electron micrographs of a gap junction (gj; brackets) between two PV + axon terminals (Pt). The gap junction, followed through four serial sections, measured $0.46 \mu \mathrm{m}$ in length. Both labeled terminals synapse onto the same PV-negative perikaryon of a pyramidal cell (arrows; Upk). Scale bar, $0.5 \mu \mathrm{m}$.

2000; Woodson et al., 2000; McDonald and Betette, 2001), closer light microscopic examination in the present study revealed that there was a small number of lightly stained spines associated with dendrites and perikarya. Likewise, spines are evident in photomicrographs and drawings of PV + neurons in the primate basolateral amygdala (Pitkänen and Amaral, 1993).

\section{Gap junctions between PV+ dendrites}

The ultrastructure of gap junctions was easiest to appreciate in preparations labeled for PV immunoreactivity with IGS and V-VIP techniques, because these methods yielded a granular reaction product (Fig. $2 A, B$ ) that did not obscure the heptalaminar structure of these junctions. Examples from these preparations showed convergence of the plasma membranes of adjacent dendrites, with their double leaflets lined up to form four electron- dense bands and the outer leaflets delineating a narrow electron-lucent gap (Fig. $2 A, B)$. The semidense undercoatings along the inner leaflets of the junctional apposition were also apparent. However, even in the presence of intense IP immunoreactivity, the unique spacing of the inner and outer leaflets and intercalated central gap were still recognizable, especially when compared with neighboring chemical synapses (Fig. 2C,D). As described previously (Fukuda and Kosaka, 2000, 2003), peroxidase reaction product often filled the gap (Figs. 2C,D, 3). Overall, 14 of the $18 \mathrm{PV}+$ dendrodendritic gap junctions were found in IP-labeled preparations and two each from material processed for PV immunoreactivity using the IGS and V-VIP techniques.

Although most dendrodendritic gap junctions were $0.2-0.5 \mu \mathrm{m}$ in length, the three largest were between 0.9 and $1.5 \mu \mathrm{m}$ (Figs. 3, 5C). The paired dendrites were $0.2-1.7 \mu \mathrm{m}$ in diameter but were generally of small to medium caliber (Fig. $5 B$ ). As summarized in a scatter plot (Fig. 5A), approximately as many dendritic pairs were similar in diameter (Fig. $2 A, C, D$ ) as disparate in size (Figs. $2 B, 3,7 A, B$ ).

\section{Gap junctions between PV+ axon terminals}

Six pairs of $\mathrm{PV}+$ terminals were found to form gap junctions in IP-labeled preparations (Figs. 4, 5C). Even in densely labeled profiles, axon terminals could be distinguished from dendrites by their characteristic aggregation of synaptic vesicles (Fig. 4). In four of six cases, one or both of the $\mathrm{PV}+$ terminals appeared to make synaptic contact onto pyramidal neuronal perikarya (Fig. 4), and a possible fifth instance had a pyramidal cell very close to one of the PV + terminals, but not all serial sections were available. These gap junctions measured between 0.2 and $0.46 \mu \mathrm{m}$ in diameter (Fig. 5C, white bars), which is within the same range as most of the dendrodendritic gap junctions described above. Examination of serial sections revealed that the axon terminals involved in the gap junctions were not adjacent terminals of the same axonal branch. Thus, it is likely that each terminal belongs to the axon of a different $\mathrm{PV}+$ interneuron.

\section{Chemical synapses and gap junctions between PV+ neurons:} a quantitative survey

In addition to connections via gap junctions, $\mathrm{PV}+$ neurons were also found to be interconnected by chemical synapses (Figs. 6-9). To determine the relative proportions of these two types of contacts, a quantitative survey that pooled samples from two experiments collected 664 chemical synapses onto PV + neurons, of which 130 (19.6\%) were from PV + terminals (Table 1). In one sample, a sagittal section from a preparation fixed with $2 \%$ para- 
formaldehyde $/ 2.5 \%$ glutaraldehyde (Fig. $1 B), 79$ of 425 synapses $(18.6 \%)$ onto $\mathrm{PV}+$ neurons were from $\mathrm{PV}+$ terminals. From the other sample, a coronal section from a preparation fixed with acrolein/ paraformaldehyde, 51 of 239 synapses (21.3\%) onto PV+ neurons were from $\mathrm{PV}+$ terminals. All synapses formed by $\mathrm{PV}+$ terminals were symmetrical. The proportions of $\mathrm{PV}+$ synapses targeting the various compartments of PV + neurons is summarized in Table 1 and discussed in more detail below.

Of the $18 \mathrm{PV}+$ dendrodendritic gap junctions observed, seven were found in the quantitative survey: four from the first sample and three from the second. In the overall survey, gap junctions were seen at 7 of 17 dendrodendritic juxtapositions (41.2\%): 4 of 10 from the first sample and 3 of 7 from the second. In addition, one of the six gap junctions described between $\mathrm{PV}+$ axon terminals was found in the quantitative survey. Thus, PV + neurons received $130 \mathrm{PV}+$ chemical synapses and formed eight gap junctions with $\mathrm{PV}+$ profiles, a ratio of $\sim 16: 1$.

Chemical synapses onto $\mathrm{PV}+$ perikarya Synaptic inputs to $16 \mathrm{PV}+$ perikarya were counted as part of the quantitative survey. Of the 16 perikarya, 12 were solidly labeled with a uniformly electron-dense reaction product (Fig. $6 A-C$ ), whereas four appeared less densely labeled, with a fine granular reaction product (Fig. 6D). Of the 163 synapses contacting these $\mathrm{PV}+$ perikarya (Table 1), approximately one-third were from $\mathrm{PV}+$ terminals $(30.1 \%$; 49 of 163). More than three-fourths $(78 \% ; 111$ of 143$)$ of classified synapses onto $\mathrm{PV}+$ perikarya were symmetrical, and approximately half of these $(44 \% ; 49$ of 111) were formed by PV + axon terminals.

Although a large majority of the $\mathrm{PV}+$ terminals innervating $\mathrm{PV}+$ perikarya were small to medium sized (Figs. 5D, 6A, C,D, center right), several were larger, with areas $>1 \mu \mathrm{m}^{2}$ (Figs. $5 D$, $6 B, D$, bottom left). Our analysis of axon terminal size in light microscopic studies suggests that the latter may arise from PV+ projection neurons of the basal forebrain (see Materials and Methods). Thus, in animals with PHA-L injections into the basal forebrain, 97\% (33 of 34) of PHA-L-labeled axonal varicosities contacting $\mathrm{PV}+$ perikarya in the BLa (i.e., terminals presumed to arise from $\mathrm{PV}+$ projection neurons in the basal forebrain) had cross-sectional areas $>1 \mu \mathrm{m}^{2}$ (mean $\left.\pm \mathrm{SD}, 2.54 \pm 1.47 \mu \mathrm{m}^{2}\right)$. In contrast, $100 \%$ (95 of 95) of PV + axonal varicosities contacting unlabeled pyramidal perikarya in the BLa (i.e., terminals presumed to arise from PV + interneurons) had cross-sectional areas $<1.0 \mu \mathrm{m}^{2}$ (mean $\left.\pm \mathrm{SD}, 0.37 \pm 0.16 \mu \mathrm{m}^{2}\right)$. These approximate comparisons suggest that $\sim 10 \%$ (5 of 49 ) (Fig. $5 D$ ) of PV+ terminals synapsing with $\mathrm{PV}+$ perikarya in our quantitative electron microscopic survey may actually represent PV + basal forebrain afferents. Because the six pairs of axon terminals with gap junctions between them (Figs. 4, 5C, white bars) all had crosssectional areas $<1.0 \mu \mathrm{m}^{2}$, they appear to represent axons of PV+ interneurons.

Of the five larger PV + terminals found in the quantitative
B
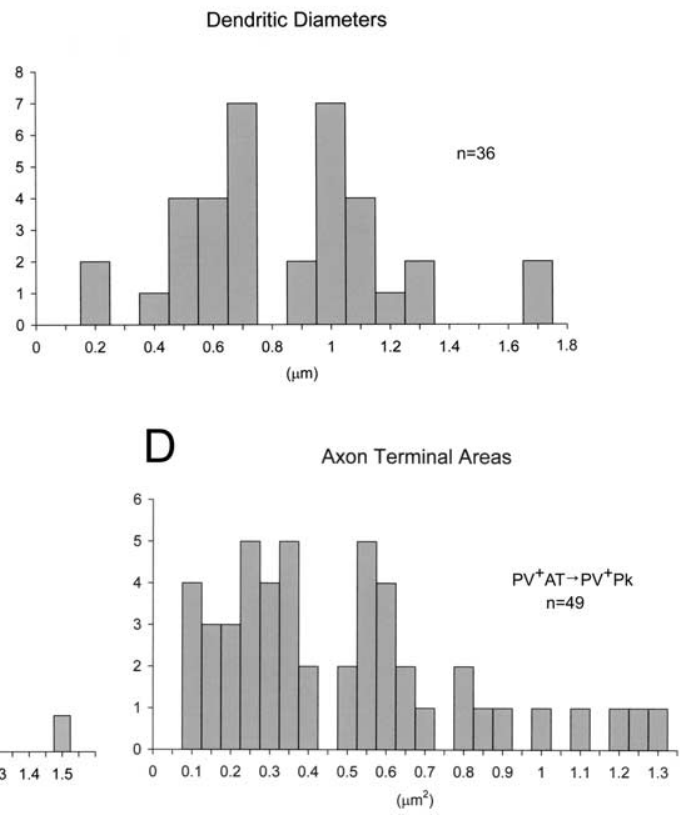

$(\mu \mathrm{m})$ $\begin{array}{llllllll}0.9 & 1 & 1.1 & 1.2 & 1.3 & 1.4 & 1.5\end{array}$ $\left(\mu \mathrm{m}^{2}\right)$

Figure 5. Graphic representation of measured profiles. $A$, A scatter plot of the diameters of the 18 pairs of PV + dendrites in the 列 $n=6)$. All junctions but one were followed, at least partially, through serial sections. $\boldsymbol{D}$, Histogram of the areas of $49 \mathrm{PV}+$ $16 \mathrm{PV}+$ perikarya of the quantitative survey.

survey to innervate $\mathrm{PV}+$ perikarya, three were also seen to make synaptic contact with pyramidal neuronal perikarya (Fig. $6 B, D$, bottom left). In addition, a PV + axon initial segment was found to receive synaptic input from at least five PV + terminals (Fig. $6 E$ ) in a sample that was not part of the quantitative survey; one of these terminals (Fig. 6E, asterisk) was of similar size to the larger terminals described and made synaptic contact with the proximal dendrite of a pyramidal neuron, as well as with the PV+ axon initial segment.

\section{Chemical synapses onto PV + dendrites}

Overall, $15.1 \%$ (68 of 450 ) of the synaptic contacts onto PV+ dendrites were from PV + terminals. The proportion was somewhat higher for larger-caliber dendrites (16.8\%) than for smallercaliber dendrites (13.8\%) (Table 1). Approximately one-half $(44.7 \% ; 174$ of 389$)$ of classified synapses onto PV+ dendrites were symmetrical, and $39 \%$ of these (68 of 174) were formed by $\mathrm{PV}+$ axon terminals. Although many short PV + dendritic profiles surveyed had synaptic inputs only from unlabeled terminals, a number of more extended views of PV + dendrites showed synaptic inputs from several PV + terminals in close proximity (Fig. $7 A-C$ ). The frequency and availability of these extended views of $\mathrm{PV}+$ dendritic trunks and branches were limited by penetration of the antibody after fixation for electron microscopy, combined with the lack of coherent stratification of PV+ dendritic arbors in the rat BLa. For three of the seven PV + dendrodendritic gap junctions found in the quantitative survey, the nearest PV + chemical synapses to the paired dendrites were located. In two cases, one of which is shown in Figure 3, the PV+ chemical synapses were in very close proximity to the gap junction. In one more extended view of a PV + dendrite, chemical synaptic inputs from three PV + terminals were found between 9 

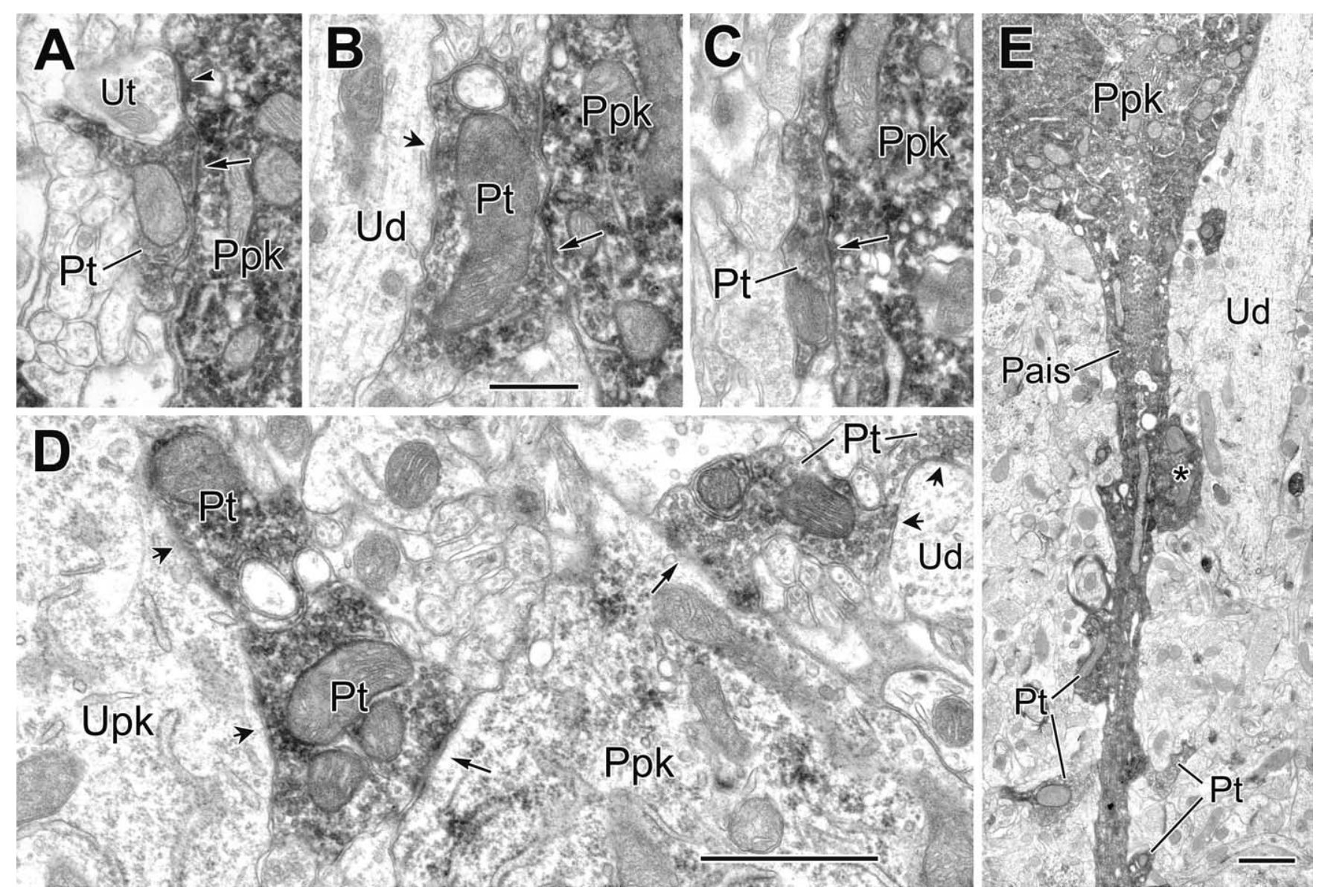

Figure 6. Electron micrographs of synapses from PV + terminals onto PV + perikarya and an axon initial segment. $A, A P V+$ perikaryon (Ppk) receives synaptic input from a PV + terminal (Pt; arrow) and asymmetrical synaptic contact from an adjacent unlabeled terminal (Ut; arrowhead). $\boldsymbol{B}, \boldsymbol{C}$, Examples of two PV + terminals (Pt; arrows) synapsing onto the same PV + perikaryon (Ppk). In $\boldsymbol{B}$, the larger PV + terminal also makes synaptic contact onto an unlabeled proximal dendrite of a pyramidal cell (Ud; short arrow). $\boldsymbol{D}$, Two PV + terminals make synaptic contact onto the same moderately labeled PV + perikaryon (Ppk) that was originally identified at the light microscopic level (long arrows). The larger PV + terminal at the left is also one of two adjacent PV + terminals synapsing onto an unlabeled perikaryon of a pyramidal (Upk; short arrows). At the right, the smaller PV + terminal is one of two PV + terminals making synaptic contact with the same unlabeled dendrite (Ud; short arrows). E, Axon initial segment (Pais) arising from a PV + perikaryon receives synaptic contact from four small (Pt) and one large PV + terminal (asterisk). The latter also makes synaptic contact with a large-caliber PV-negative dendrite (Ud). Scale bars: (in $\boldsymbol{B}) \boldsymbol{A}-\boldsymbol{C}, 0.5 \mu \mathrm{m} ; \boldsymbol{D}, \boldsymbol{E}, 1 \mu \mathrm{m}$.

and $11 \mu \mathrm{m}$ from a PV+ dendrodendritic gap junction (Fig. $7 A, B)$.

\section{Chemical synapses onto PV+ spines}

Large- and small-caliber dendrites, as well as perikarya, of PV+ interneurons can give rise to spines and spine-like cytoplasmic extensions. Spines of $\mathrm{PV}+$ interneurons received synaptic input from both unlabeled terminals (Fig. $9 D, G$ ) and PV+ terminals (Fig. 9E-G) (Table 1). Of the 51 synapses contacting these PV+ spines (Table 1), one-fourth were from PV + terminals (25\%; 13 of 51). Approximately half (54\%; 24 of 44) of classified synapses onto PV + spines were symmetrical, and approximately half of these (54\%; 13 of 24) were formed by PV+ axon terminals. Multiple synaptic inputs to PV + spines were common. Some PV+ spines received synaptic input from both labeled and unlabeled terminals (Fig. $9 F, G$ ), whereas others received pairs of asymmetrical contacts, sometimes opposite one another, with subsynaptic dense bodies (Fig. 9A).

\section{Discussion}

This study demonstrates that the basolateral amygdala contains a network of PV+ interneurons coupled by electrical and chemical synapses. This is the first study to describe dendrodendritic gap junctions interconnecting PV+ interneurons in the basolateral amygdala. It also provides the first documentation in the mammalian forebrain of gap junctions between interneuronal axon terminals.

\section{Chemical synapses onto PV+ interneurons}

Approximately half of the classified synapses onto PV + neurons were symmetrical (54\%; 309 of 576), with the proportions varying in different compartments (perikarya, 78\%, 111 of 143; dendrites, $45 \%, 174$ of 389 ; spines, $55 \%$, 24 of 44 ). The majority of these symmetrical synapses are probably GABAergic inhibitory synapses formed by local interneurons (Carlsen, 1988; McDonald et al., 2002; Muller et al., 2003). The remaining classified synapses $(46 \%$; 267 of 576$)$ onto PV+ neurons were asymmetrical. Most of these synapses are provided by collaterals of local glutamatergic pyramidal neurons and glutamatergic projections from limbic association cortices and intralaminar thalamic nuclei (Smith et al., 2000; Woodson et al., 2000).

Twenty percent (130 of 664) of all synapses onto PV+ neurons were PV + synapses, and these comprised $41-54 \%$ of all symmetrical synapses depending on the postsynaptic compartment (perikarya, $44 \%$; dendrites, $41 \%$; spines, $54 \%$ ). Perikarya exhibited the greatest incidence of PV+ synapses (30\%; 49 of 163 


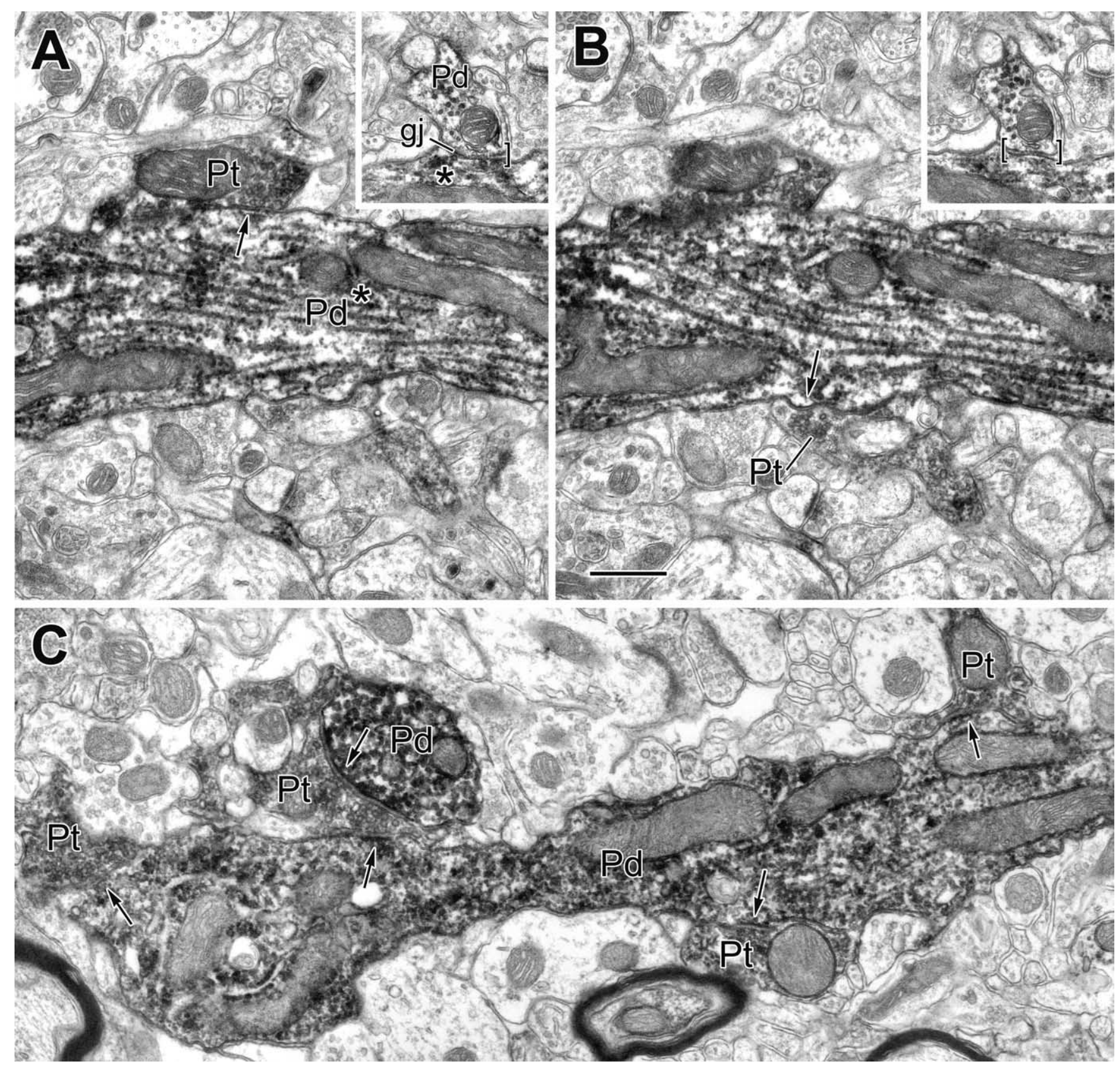

Figure 7. Electron micrographs of PV + dendrites exhibiting a gap junction and receiving synapses from PV + terminals. $A, B$, Two serial sections of a large-caliber PV + dendrite (Pd) showing two of three PV + terminals (Pt; arrows) that make synaptic contact with this dendrite. The inset depicts a gap junction (gj) involving this dendrite and a small PV + dendrite (Pd; brackets) that was located $11 \mu \mathrm{m}$ from the synapses. The asterisks indicate that all contacts are with the same PV + dendritic trunk. C, Longitudinal view of a varicose PV + dendrite (Pd) receiving synaptic input from four PV + terminals (Pt; arrows). One of these PV + terminals also synapses onto an adjacent PV + dendritic cross section (Pd; arrow; top left). Scale bar, $0.5 \mu \mathrm{m}$.

of total synapses), with lower percentages associated with dendrites $(15 \% ; 68$ of 450$)$ and spines $(25 \% ; 13$ of 51$)$. Although our measurements of the sizes of PV + terminals in contact with the perisomatic domain of PV+ neurons suggest that some large $\mathrm{PV}+$ axon terminals may originate from $\mathrm{PV}+$ projection neurons of the basal forebrain (see Materials and Methods), the great majority of $\mathrm{PV}+$ terminals are probably axons of $\mathrm{PV}+$ interneurons in the BLa.

These results differ from those obtained in the cat basolateral amygdala, in which asymmetrical synapses predominated in all $\mathrm{PV}+$ postsynaptic compartments, and no PV-to-PV synapses were reported (Smith et al., 1998). This may be a species difference, because many contacts of $\mathrm{PV}+$ terminals with $\mathrm{PV}+$ perikarya and proximal dendrites were seen at the light microscopic level in the monkey and human basolateral amygdala (Pitkänen and Amaral, 1993; Sorvari et al., 1995), and recent electrophysiological studies have found evidence for synapses between pairs of PV + interneurons in the mouse BLa (Woodruff and Sah, 2003).

The innervation of $\mathrm{PV}+$ neurons by $\mathrm{PV}+$ terminals in the BLa is similar to that seen in the cortex. Thus, in field CA1 of the hippocampus, symmetrical synapses formed by GABA+ and $\mathrm{GABA}+/ \mathrm{PV}+$ terminals are concentrated on the perikarya of $\mathrm{PV}+$ interneurons (Gulyas et al., 1999). Neocortical basket cells, many of which are $\mathrm{PV}+$, provide a largely perisomatic innervation of other basket cells (Tamas et al., 1998). There is also elec- 

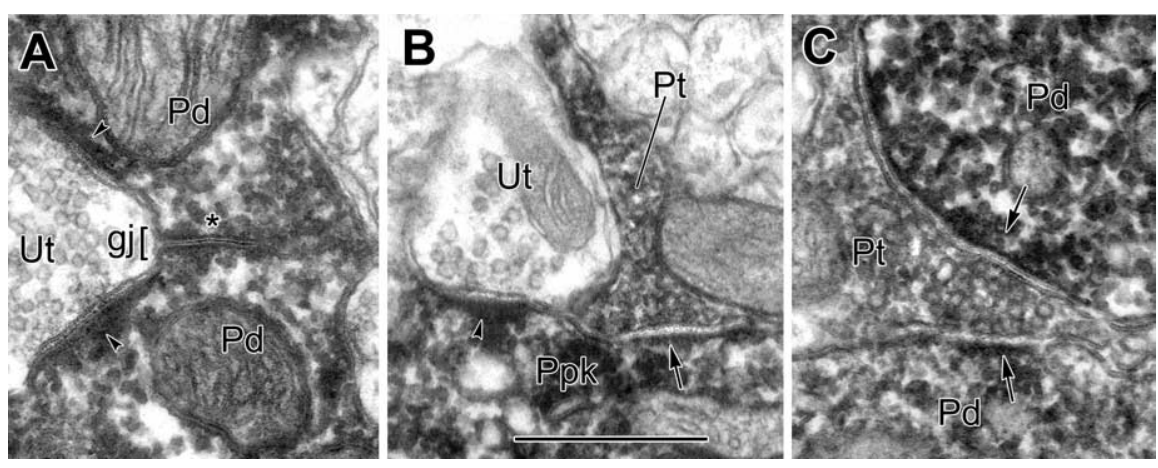

Figure 8. Comparison of the morphology of an IP-labeled gap junction with IP-labeled chemical synapses. $A$, Detail of the IP-labeled PV + dendrites (Pd) shown in Figure 2C in gap junctional contact (gj; asterisk). Each dendrite receives an asymmetrical synapse (arrowheads) from an unlabeled terminal (Ut). B, Detail from Figure $6 A$ of an IP-labeled PV + perikaryon (Ppk) receiving synaptic contacts from a PV + axon terminal (Pt; arrow) and unlabeled axon terminal (Ut; arrowhead). C, Detail from Figure 7C of an IP-labeled PV + terminal (Pt) making synaptic contacts (arrows) with two PV + dendrites (Pd). Note that peroxidase reaction product fills the central gap of the gap junction, whereas an unstained synaptic cleft separates the outer leaflets of presynaptic and postsynaptic plasma membranes of chemical synapses. Scale bar: (in $\boldsymbol{B}) \boldsymbol{A}-\boldsymbol{C}, 0.5 \mu \mathrm{m}$.

trophysiological evidence for extensive interconnections of PV+ interneurons in the neocortex (Galarreta and Hestrin, 2001).

\section{Gap junctions connecting PV + interneurons}

Gap junctions mediate electrical coupling, which appears to be critical for synchronizing the activity of the interconnected neurons (Galarreta and Hestrin, 2001). The morphology of the gap junctions observed between PV + interneurons of the BLa in the present study is identical to that of gap junctions in other brain regions, including the neocortex and hippocampus (Katsumaru et al., 1988; Peters et al., 1991; Tamas et al., 2000; Fukuda and Kosaka, 2000, 2003). Our findings are consistent with recent electrophysiological studies demonstrating electrical coupling of $\mathrm{PV}+$ neurons in the BLa (Woodruff and Sah, 2003). Likewise, there is electrophysiological evidence for gap junction-mediated electrical coupling of PV + interneurons in the cortex (Gibson et al., 1999; Venance et al., 2000; Blatow et al., 2003).

Most of the dendrodendritic gap junctions in the BLa were similar in size to those of the neocortex $(0.2-0.5 \mu \mathrm{m}$ in diameter $)$ (Fukuda and Kosaka, 2003), but a few were larger $(0.9-1.5 \mu \mathrm{m}$ in diameter). As in other brain areas, it appears that connexin 36 (Cx 36) may be the main protein forming connexons of neuronal gap junctions in the amygdala (Belluardo et al., 2000; Rash et al., 2004). Using data on connexon density and conductance, it is possible to calculate a maximal conductance for individual gap junctions (Fukuda and Kosaka, 2003). Assuming a packing density of 4000-10,000 connexons/ $\mu \mathrm{m}^{2}$ (Tuttle et al., 1986; Baldridge et al., 1987) and a unitary conductance of $14 \mathrm{pS}$ for single Cx 36 connexons (Teubner et al., 2000), the conductance of the average dendrodendritic gap junction in the BLa $(0.45 \pm$ $0.36 \mu \mathrm{m}$ in diameter; mean $\pm \mathrm{SD}$ ) could be as high as $8.9-22.4$ $\mathrm{nS}$. Because of the larger mean size of gap junctions in the BLa compared with the neocortex $(0.22 \pm 0.09 \mu \mathrm{m})$, these conductances are higher than those calculated for the latter (Fukuda and Kosaka, 2003). Although these calculated conductances are both much higher than those obtained in electrophysiological studies in the cortex (Gibson et al., 1999; Galarreta and Hestrin, 1999, 2001), it appears that gating of gap junctions and electrotonic attenuation of gap junctional potentials with somatic recordings may contribute to the lower values obtained experimentally.

The location of dendrodendritic gap junctions, as well as their density, may determine their ability to influence the firing of neurons versus participation in purely local interactions. As in the cerebral cortex (Sloper and Powell, 1978; Fukuda and Kosaka, 2003), most of the gap junctions seen in the BLa in the present study were associated with dendrites of intermediate thickness (0.5-1.5 $\mu \mathrm{m}$ thick). These dendrites mainly correspond to secondary and more distal dendrites of PV + neurons in the BLa. Although the present study could not determine the number of gap junctions exhibited by individual PV+ neurons, it has been estimated that there may be an average of $5.3 \pm 2.3$ per $\mathrm{PV}+$ neuron in the neocortex (Fukuda and Kosaka, 2003). Because some cortical interneurons exhibit dendritic action potentials (Martina et al., 2000), it is possible that gap junctional potentials could be actively propagated to interneuronal somata.

This study includes the first report of morphologically identified gap junctions between axon terminals in the mammalian forebrain, although there is indirect evidence for gap junctions interconnecting more proximal portions of pyramidal cell axons in the cortex (Schmitz et al., 2001). There have been no reports of interneuronal axonal gap junctions in the cortex, but recent electrophysiological studies and computer simulations have provided evidence for their existence in this region (Traub et al., 2001; Fisahn et al., 2004). The latter studies suggest that spikes can cross through interneuronal axonal gap junctions and synchronize GABA release from adjacent PV + terminals. These spikes could also be conducted antidromically and invade the soma, and perhaps other axonal branches, of the coupled interneuron. These mechanisms would be expected to synchronize the activity of coupled PV+ interneuronal populations (Traub et al., 2001).

\section{Functional implications}

Although little is known about the network properties of PV + neurons in the BLa, their close resemblance to fast-spiking PV+ interneurons in the neocortex and hippocampus suggests that they may exhibit many functional similarities. As in the cortex, it seems probable that networks of interconnected $\mathrm{PV}+$ neurons in the BLa may be critical for the generation of rhythmic oscillations in the gamma range $(30-100 \mathrm{~Hz})$. Gamma oscillations, which have also been observed in the BLa (Collins et al., 2001), appear to be important for providing a temporal context, relative to which specific information is encoded (Singer, 1993; Freund and Buzsáki, 1996).

There is evidence that both electrical synapses and GABAergic chemical synapses among PV + neurons contribute to gamma oscillations in the cortex. Electrophysiological and computer simulation studies indicate that gamma oscillations are an emergent property of networks of fast-spiking interneurons interconnected by chemical synapses using $\mathrm{GABA}_{\mathrm{A}}$ receptors (Whittington et al., 1995; Jefferys et al., 1996). The decay constant of the GABAergic synapses determines the exact frequency of the oscillation (Whittington et al., 1995). In the BLa, as in the cortex, PV+ neurons exhibit high levels of the $\alpha 1$ subunit of the $\mathrm{GABA}_{\mathrm{A}}$ receptor (McDonald and Mascagni, 2004). The fast kinetics of these receptors may be important for promoting temporal precision in the oscillations of the $\mathrm{PV}+$ interneuronal network (Klausberger et al., 2002).

Dendritic electrical synapses, as well as the fast kinetics of the GABAergic chemical synapses, stabilize and increase synchrony of 


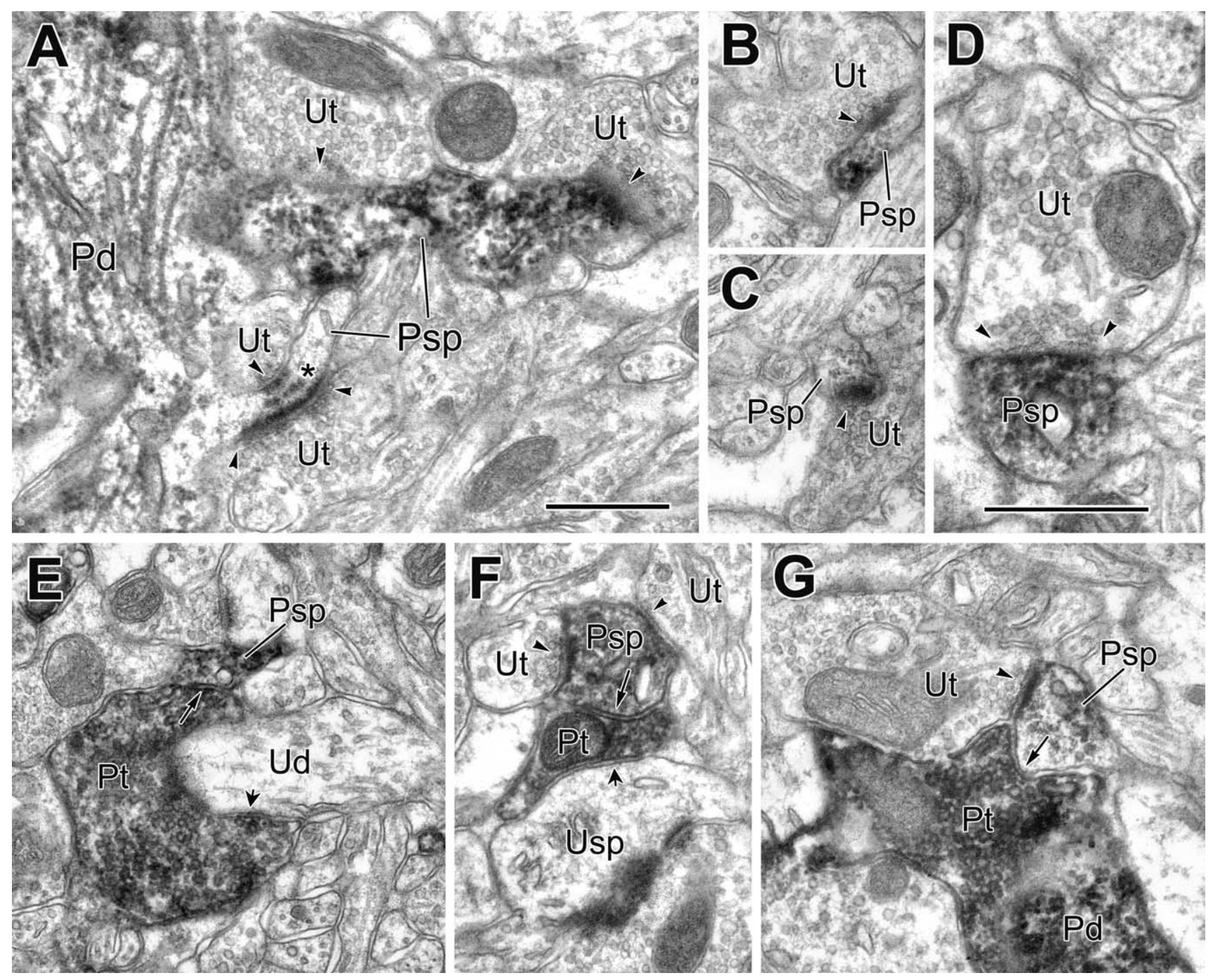

Figure 9. Electron micrographs of PV + spines receiving synaptic input from both PV-negative and PV + terminals. $A$, Two spines (Psp) emerge from a PV + dendrite (Pd), each receiving synaptic input from two unlabeled terminals (Ut; arrowheads). In serial sections, all contacts appeared asymmetrical. One of the spines has subsynaptic dense bodies (asterisk). B, C, Two neighboring PV+ spines (Psp) receive synaptic input from unlabeled terminals (Ut; arrowheads). D, A higher-magnification view of a PV + spine (Psp) receiving unlabeled synaptic input (Ut; arrowheads). $\boldsymbol{E}, \mathrm{A}$ PV + spine (Psp) receives synaptic input from a PV + terminal (Pt; long arrow) that is also wrapped around and synapsing onto an unlabeled dendrite (Ud; short arrow). $\boldsymbol{F}, \mathrm{A}$ PV + spine (Psp) receives synaptic input from a PV + terminal (Pt; long arrow) and two unlabeled terminals (Ut; arrowheads). The PV + terminal also makes synaptic contact onto an unlabeled spine (Usp; short arrow). G, $\mathrm{APV}+$ spine (Psp) receives synaptic input from PV+ (Pt; arrow) and unlabeled (Ut; arrowhead) terminals. The PV+ terminal appears to be contacting a PV+ dendrite (Pd). $\boldsymbol{A}-\mathbf{C}$ and $\boldsymbol{E}-\mathbf{G}$ are the same magnification. Scale bars, $0.5 \mu \mathrm{m}$.

gamma oscillations in cortical interneuronal networks (Deans et al., 2001; Hormuzdi et al., 2001; Traub et al., 2001; Bartos et al., 2002; Buhl et al., 2003). This increase in synchrony may arise from synergistic local interactions of electrical and GABAergic chemical synapses, in which the IPSPs generated by chemical synapses curtail the excitatory gap junction potentials of the electrical synapses (Tamas et al., 2000). The close proximity of many of the PV-PV chemical synapses and gap junctions in the present study (Fig. 3) suggests that a similar mechanism may exist in the BLa. Our finding that the ratio of PV+ chemical synapses to gap junctions was $\sim 16: 1$ is consistent with recent electrophysiological studies, which found a predominance of $\mathrm{GABA}_{\mathrm{A}}$ receptor-mediated inhibition in all electrically coupled pairs of $\mathrm{PV}+$ interneurons in mouse BLa (P. Sah and A. R. Woodruff, personal communication).
Table 1. PV-positive postsynaptic targets of PV-positive and PV-negative axon terminals forming symmetrical, asymmetrical, or unclassified synapses

\begin{tabular}{lcccr}
\hline ATs/synapses & PV + perikarya & $\begin{array}{c}\text { Large-caliber PV+ dendrites } \\
(>1 \mu \mathrm{m})\end{array}$ & $\begin{array}{c}\text { Small-caliber PV+ dendrites } \\
(<1 \mu \mathrm{m})\end{array}$ & PV+ spines \\
\hline PV+/Sym & $49(30.1 \%)$ & $33(16.8 \%)$ & $35(13.8 \%)$ & $13(25.5 \%)$ \\
PV-/Sym & $62(38.0 \%)$ & $48(24.5 \%)$ & $58(22.8 \%)$ & $11(21.6 \%)$ \\
PV-/Asym & $32(19.6 \%)$ & $90(45.9 \%)$ & $125(49.2 \%)$ & $20(39.2 \%)$ \\
PV-/UC & $20(12.4 \%)$ & $25(12.8 \%)$ & $36(14.2 \%)$ & $7(13.7 \%)$ \\
Total & $163(100 \%)$ & $196(100 \%)$ & $254(100 \%)$ & $51(100 \%)$ \\
\hline
\end{tabular}

Counts are pooled from two samples. Some axon terminals formed more than one synapse. ATs, Axon terminals; Sym, symmetrical synapse; Asym, asymmetrical synapse; UC, unclassifed synapse (i.e., not clearly symmetrical or asymmetrical).

\section{References}

Aggleton JP (1992) The amygdala: neurobiological aspects of emotion, memory, and mental dysfunction. New York: Wiley.

Aggleton JP (2000) The amygdala: a functional analysis. New York: Wiley. Baldridge WH, Ball AK, Miller RG (1987) Dopaminergic regulation of horizontal cell gap junction particle density in goldfish retina. J Comp Neurol 265:428-436. 
Bartos M, Vida I, Frotscher M, Meyer A, Monyer H, Geiger JR, Jonas P (2002) Fast synaptic inhibition promotes synchronized gamma oscillations in hippocampal interneuron networks. Proc Natl Acad Sci USA 99:13222-13227.

Belluardo N, Mudò G, Trovato-Salinaro A, Gurun SL, Charollais A, SerreBeinier V, Amato G, Haefliger J-A, Meda P, Condorelli DF (2000) Expression of connexin 36 in the adult and developing rat brain. Brain Res 865:121-138.

Blatow M, Rozov A, Katona I, Hormuzdi SG, Meyer AH, Whittington MA, Caputi A, Monyer H (2003) A novel network of multipolar bursting interneurons generates theta frequency oscillations in neocortex. Neuron 38:805-817.

Buhl DL, Harris K, Hormuzdi S, Monyer H, Buzsáki G (2003) Selective impairment of hippocampal gamma oscillations in connexin-36 knockout mouse in vivo. J Neurosci 23:1013-1018.

Buzsaki G (2002) Theta oscillations in the hippocampus. Neuron $33: 325-340$.

Carlsen J (1988) Immunocytochemical localization of glutamate decarboxylase in the rat basolateral amygdaloid nucleus, with special reference to GABAergic innervation of amygdalostriatal projection neurons. J Comp Neurol 273:513-526.

Carlsen J, Heimer L (1986) A correlated light and electron microscopic immunocytochemical study of cholinergic terminals and neurons in the rat amygdaloid body with special emphasis on the basolateral amygdaloid nucleus. J Comp Neurol 244:121-136.

Collins DR, Pelletier JG, Paré D (2001) Slow and fast (gamma) neuronal oscillations in the perirhinal cortex and lateral amygdala. J Neurophysiol 85:1661-1672.

Conde F, Lund JS, Jacobowitz DM, Baimbridge KG, Lewis DA (1994) Local circuit neurons immunoreactive for calretinin, calbindin D-28k or parvalbumin in monkey prefrontal cortex: distribution and morphology. J Comp Neurol 341:95-116.

Deans MR, Gibson JR, Sellitto C, Connors BW, Paul DL (2001) Synchronous activity of inhibitory networks in neocortex requires electrical synapses containing connexin36. Neuron 31:477-485.

Fisahn A, Contractor A, Traub RD, Buhl EH, Heinemann SF, McBain CJ (2004) Distinct roles for the kainate receptor subunits GluR5 and GluR6 in kainate-induced hippocampal gamma oscillations. J Neurosci 24:9658-9668.

Freund TF (1989) GABAergic septohippocampal neurons contain parvalbumin. Brain Res 478:375-381.

Freund TF, Buzsáki G (1996) Interneurons of the hippocampus. Hippocampus 6:347-470.

Fukuda T, Kosaka T (2000) Gap junctions linking the dendritic network of GABAergic interneurons in the hippocampus. J Neurosci 20:1519-1528.

Fukuda T, Kosaka T (2003) Ultrastructural study of gap junctions between dendrites of parvalbumin-containing GABAergic neurons in various neocortical areas of the adult rat. Neuroscience 120:5-20.

Galarreta M, Hestrin S (1999) Network of fast-spiking cells in the neocortex connected by electrical synapses. Nature 40272-40275.

Galarreta M, Hestrin S (2001) Electrical synapses between GABA-releasing interneurons. Nat Rev Neurosci 2:425-433.

Galarreta M, Erdelyi F, Szabo G, Hestrin S (2004) Electrical coupling among irregular-spiking GABAergic interneurons expressing cannabinoid receptors. J Neurosci 24:9770-9778.

Gibson JR, Beierlein M, Connors BW (1999) Two networks of electrically coupled inhibitory neurons in neocortex. Nature 402:75-79.

Gulyas AI, Megias M, Emri Z, Freund TF (1999) Total number and ratio of excitatory and inhibitory synapses converging onto single interneurons of different types in the CAl area of the rat hippocampus. J Neurosci 19:10082-10097.

Hormuzdi SG, Pais I, LeBeau FE, Towers SK, Rozov A, Buhl EH, Whittington MA, Monyer H (2001) Impaired electrical signaling disrupts gamma frequency oscillations in connexin 36-deficient mice. Neuron 31:487-495.

Jefferys JG, Traub RD, Whittington MA (1996) Neuronal networks for induced " $40 \mathrm{~Hz}$ " rhythms. Trends Neurosci 19:202-208.

Katsumaru H, Kosaka T, Heizmann CW, Hama K (1988) Gap junctions on GABAergic neurons containing the calcium-binding protein parvalbu$\min$ in the rat hippocampus (CA1 region). Exp Brain Res 72:363-370.

Kemppainen S, Pitkänen A (2000) Distribution of parvalbumin, calretinin, and calbindin- $\mathrm{D}_{28 \mathrm{k}}$ immunoreactivity in the rat amygdaloid complex and colocalization with gamma-aminobutyric acid. J Comp Neurol 426:441-467.

Klausberger T, Roberts JD, Somogyi P (2002) Cell type- and input-specific differences in the number and subtypes of synaptic $\mathrm{GABA}_{\mathrm{A}}$ receptors in the hippocampus. J Neurosci 22:2513-2521.

Lanciego JL, Goede PH, Witter MP, Wouterlood FG (1997) Use of peroxidase substrate vector VIP for multiple staining in light microscopy. J Neurosci Methods 74:1-7.

Martina M, Vida I, Jonas P (2000) Distal initiation and active propagation of action potentials in interneuron dendrites. Science 287:295-300.

McDonald AJ (1992) Cell types and intrinsic connections of the amygdala. In: The amygdala (Aggleton JP, ed), pp 67-96. New York: Wiley.

McDonald AJ, Betette R (2001) Parvalbumin containing neurons in the rat basolateral amygdala: morphology and colocalization of calbindin D-28k. Neuroscience 102:413-425.

McDonald AJ, Mascagni F (1997) Projections of the lateral entorhinal cortex to the amygdala: a Phaseolus vulgaris leucoagglutinin study in the rat. Neuroscience 77:445-459.

McDonald AJ, Mascagni F (2004) Parvalbumin-containing interneurons in the basolateral amygdala express high levels of the alphal subunit of the $\mathrm{GABA}_{\mathrm{A}}$ receptor. J Comp Neurol 473:137-146.

Mello LE, Tan AM, Finch DM (1992) GABAergic synaptic transmission in projections from the basal forebrain and hippocampal formation to the amygdala: an in vivo iontophoretic study. Brain Res 587:41-48.

Muller JF, Mascagni F, McDonald AJ (2003) Synaptic connections of distinct interneuronal subpopulations in the rat basolateral amygdalar nucleus. J Comp Neurol 456:217-236.

Oya H, Kawasaki H, Howard III MA, Adolphs R (2002) Electrophysiological responses in the human amygdala discriminate emotion categories of complex visual stimuli. J Neurosci 22:9502-9512.

Paré D, Collins DR (2000) Neuronal correlates of fear in the lateral amygdala: multiple extracellular recordings in conscious cats. J Neurosci 20:2701-2710.

Paré D, Gaudreau H (1996) Projection cells and interneurons of the lateral and basolateral amygdala: distinct firing patterns and differential relation to theta and delta rhythms in conscious cats. J Neurosci 16:3334-3350.

Paré D, Collins DR, Pelletier JG (2002) Amygdala oscillations and the consolidation of emotional memories. Trends Cogn Sci 6:306-314.

Paxinos G, Watson C (1986) The rat brain in stereotaxic coordinates. New York: Academic.

Peters A, Palay SL, Webster HD (1991) The fine structure of the nervous system. New York: Oxford UP.

Pitkänen A, Amaral DG (1993) Distribution of parvalbumin-immunoreactive cells and fibers in the monkey temporal lobe: the amygdaloid complex. J Comp Neurol 331:14-36.

Ponomarenko AA, Korotkova TM, Haas HL (2003) High frequency (200 $\mathrm{Hz}$ ) oscillations and firing patterns in the basolateral amygdala and dorsal endopiriform nucleus of the behaving rat. Behav Brain Res 141:123-129.

Rash JE, Pereda A, Kamasawa N, Furman CS, Yasumura T, Davidson KG, Dudek FE, Olson C, Li X, Nagy JI (2004) High-resolution proteomic mapping in the vertebrate central nervous system: close proximity of connexin 35 to NMDA glutamate receptor clusters and co-localization of connexin 36 with immunoreactivity for zonula occludens protein-1 (ZO1). J Neurocytol 33:131-151.

Schmitz D, Schuchmann S, Fisahn A, Draguhn A, Buhl EH, Petrasch-Parwez E, Dermietzel R, Heinemann U, Traub RD (2001) Axo-axonal coupling. a novel mechanism for ultrafast neuronal communication. Neuron 31:831-840.

Shinnick-Gallagher P, Pitkanen A, Shekhar A, Cahill L (2003) The amygdala in brain function: basic and clinical approaches, Vol 985. New York: New York Academy of Sciences.

Singer W (1993) Synchronization of cortical activity and its putative role in information processing and learning. Annu Rev Physiol 55:349-374.

Sloper JJ, Powell TP (1978) Gap junctions between dendrites and somata of neurons in the primate sensori-motor cortex. Proc R Soc Lond B Biol Sci 203:39-47.

Smiley JF, Morrell F, Mesulam MM (1997) Cholinergic synapses in human cerebral cortex: an ultrastructural study in serial sections. Exp Neurol 144:361-368.

Smith Y, Paré J-F, Paré D (1998) Cat intraamygdaloid inhibitory network: ultrastructural organization of parvalbumin-immunoreactive elements. J Comp Neurol 391:164-179. 
Smith Y, Paré J-F, Paré D (2000) Differential innervation of paravalbuminimmunoreactive interneurons of the basolateral amygdaloid complex by cortical and intrinsic inputs. J Comp Neurol 416:496-508.

Sorvari H, Soininen H, Paljärvi L, Karkola K, Pitkänen A (1995) Distribution of parvalbumin immunoreactive cells and fibers in the human amygdaloid complex. J Comp Neurol 360:185-212.

Tamas G, Somogyi P, Buhl EH (1998) Differentially interconnected networks of GABAergic interneurons in the visual cortex of the cat. J Neurosci 18:4255-4270.

Tamas G, Buhl EH, Lorincz A, Somogyi P (2000) Proximally targeted GABAergic synapses and gap junctions synchronize cortical interneurons. Nat Neurosci 3:366-371.

Teubner B, Degen J, Sohl G, Guldenagel M, Bukauskas FF, Trexler EB, Verselis VK, De Zeeuw CI, Lee CG, Kozak CA, Petrasch-Parwez E, Dermietzel R, Willecke K (2000) Functional expression of the murine connexin 36 gene coding for a neuron-specific gap junctional protein. J Membr Biol 176:249-262.

Traub RD, Bibbig R, Piechotta A, Draguhn R, Schmitz D (2001) Synaptic and nonsynaptic contributions to giant IPSPs and ectopic spikes induced by 4 -aminopyridine in the hippocampus in vitro. J Neurophysiol 85:1246-1256

Tuttle R, Masuko S, Nakajima Y (1986) Freeze-fracture study of the large myelinated club ending synapse on the goldfish Mauthner cell: special reference to the quantitative analysis of gap junctions. J Comp Neurol 246:202-211.

Van Haeften T, Wouterlood FG (2000) Neuroanatomical tracing at high resolution. J Neurosci Methods 103:107-116.

Venance L, Rozov A, Blatow M, Burnashev N, Feldmeyer D, Monyer H (2000) Connexin expression in electrically coupled postnatal rat brain neurons. Proc Natl Acad Sci USA 97:10260-10265.

Whittington MA, Traub RD, Jefferys JG (1995) Synchronized oscillations in interneuron networks driven by metabotropic glutamate receptor activation. Nature 373:612-615.

Woodruff AR, Sah P (2003) Properties of parvalbumin-positive interneurons in the basolateral amygdala. Soc Neurosci Abstr 29:582.14.

Woodson W, Farb CR, Ledoux JE (2000) Afferents from the auditory thalamus synapse on inhibitory interneurons in the lateral nucleus of the amygdala. Synapse 38:124-137. 